\title{
Original Article \\ Outcome of tympanic membrane defect repair with fat and tetrachlorodecaoxide
}

\author{
Abeysundara U.B, Rajapaksha R.M.M.A, Prasad C.S., Wickramasinghe N.S.A., \\ Rathnayake R.A.D.I. Teaching Hospital, Anuradhapura, Sri Lanka
}

\section{Introduction}

Eardrum perforation is one of the commonest problems seen in ENT departments. Perforations can occur due to injury (barotrauma), instrumentation or following acute otitis media. Some of them heal spontaneously and some persist as perforations. Persistent perforations can restrict a patientsday-to-day activities such as swimming or having a bath. Thiscould lead middle ear infections and can be overcome by using ear plugs to a certain extent. But repair of the perforation is the permanent solution. Myringoplasty is an operation carried out to close perforations in the tympanic membrane. Various graft materials (paper-patch, fat, temporalis fascia or perichondrium) and various approaches have been used to close tympanic membrane defects and the success rates appears to be more or less similar for smaller perforations.

Ringenberg(1) first described fat plug myringoplasty in 1962, with a success rate of $87 \%$ for small perforations and thereafter fat plug myringoplasty was done worldwide with a varying success rate.

According to Kelvin Kwong, Smith and Coticchia $^{(2)}$ (2012), Fat graft myringoplasty using umbilical fat was a safe and successful

Procedure for TM repair and achieved an overall $100 \%$ rate for success in TM closure

Dursunet $\mathrm{al}^{(3)}$ (2008) added that "There was no statistically significant difference among closure rates of the perforations in the paper-patch, fat, and perichondrium myringoplasty with regard to size and these three techniques were found to be feasible for tympanic membrane perforations smaller than
3 mm.”. Konstantinidiset al (2010) ${ }^{(5)}$ concluded that fat myringoplasty achieved high success rates especially in cases of small perforations. Usage of a single fat plug is one way of closing small perforations with minimal surgical time and surgical trauma according to $\operatorname{Vikas}(2009)^{(6) .}$ There will be no post auricular wound or posterior canal wall skin elevation from the external auditory canal resulting in fewer complications and reduced operating time.

The most common technique practiced in Sri Lanka is underlay grafting, done by post aural or end aural approach. Here, the surgery is usually done under general anaesthesia. The surgeon harvests a thin fascial graft from the fascia of the temporalis muscle. The edge of the eardrum perforation is refashioned to facilitate healing. Then the posterior wall of the ear canal is elevated from its bony canal up to the middle ear. The harvested graft is placed underneath the eardrum to cover the defect and posterior canal wall is repositioned. The post auricular wound is sutured. A crape bandage is applied around the head to apply pressure over the incision site. Success rate of this surgery is up to $80 \%$.

In this method we do not perform a skin incision behind the ear. The edge of the eardrum perforation is refashioned as in routine technique. About 1 $\mathrm{cm}$ long abdominal wall incision was made to collect some fat. This harvested fat was used to fill the middle ear up to the level of ear drum. Then a small piece of gelform is placed over the eardrum and rest of the ear canal is packed with an antibiotic impregnated ear wick.

The ear pack is removed after 1 week and antibiotic ear drops were applied for 3 days 
ISSN : $2012-855 x$

Ceylon Journal of Otolaryngology

(C) 2015; College of Otorhinolaryngologists and Head and Neck Surgeons of Sri Lanka

and Oxoferin (Tetrachlorodecaoxide) drops for 1 week (Oxoferin contains fibroblast growth factor which facilitates healing). Postoperative discomfort was minimal.

\section{Method}

The study was carried out at the ENT unit at Teaching Hospital Anuradhapura 30 Patients with safe type ear drum perforations who underwent TM repair with abdominal wall fat using the described technique at ENT unit at Teaching Hospital Anuradhapura from 01/01/2014 to 31/05/2014 were included. Informed consent was obtained from each patient and confidentiality was maintained. Ethical clearance was taken from Ethical Review Committee of Faculty of Medicine, University of Rajarata. Analysis of data was done using SPSS software package.

Patients were selected the according to the selection criteria. It was made sure that the the patients were free of upper respiratory tract infection. Patients with cholesteatoma disease were excluded. Pre-operative hearing level of both ears were assessed by a PTA (pure tone audiogram). General anaesthesia (GA) was preferred mode of anaesthesia for the Surgery. Regional anaesthesia was used when the patient was not suitable for GA.

Thep pinna and the surrounding area was cleaned with two gauze pleces soaked in Betadine. Ear was reassessed under microscope regarding the suitability for surgery. The margin of the perforation was refreshed with a sharp needle. Haemostasis achievedwith a small piece of cotton wool soaked in adrenalin.

Fat was harvested from the anterior abdominal wall after cleaning with betadine. A small incision of 1 to $2 \mathrm{~cm}$ was made parallel to the skin crease with a No 15 scalpel blade. The subcutaneous fat was exposed, harvested and preserved in a gallipot containing normal saline. This incision was closed with 2 - 0 black silk which would be removed in 5-7 days period

\section{Repair the TM defect}

A piece of fat was grasped with a pair of crocodile forceps and placed it in the middle ear through the defect in the TM. Middle ear was filled with fat up to the level of TM. Excess blood and water in the middle ear was sucked out by placing a small piece of cotton wool over the TM .Gel foam was placed on the TM. External auditory meatus was packedwithTetracort (tetracycline and hydrocortisone) impregnated wick.

Post operatively patients were discharged on analgesics and dry ear precautions.

Patients were reviewed after one week and ear packs were removed. Ear was examined with aurascopeto make sure it was free of infection. Following drugs were prescribed.

1) Gentamycin ear drops 1-2 drops tds for 3days.

2) Oxoferin (Tetrachlorodecaoxide) drops 2 drops bd for 1week.

The patients were reviewed in a week and then the fifth week to assess the outcome and documentation. PTA was repeated in three months and compared with pre-operative levels.

\section{Results}

Total of 26 patients with TM defects were included in the study. Out of the 26 patients, 15 $(57.7 \%)$ were females. Mean age of the patients was 28.3 (SD 12.6) years.

Mean preoperative Pure Tone Audiogram (PTA) test results at 250, 500,1000, 2000, 4000 and $8000 \mathrm{KHz}$ in the study sample was $47,43,33$, 34 and 39 respectively. All patients showed reduction of PTA test results at least in one frequency and 12 patients showed improvement in all 6 frequencies tested. Mean difference of pre and post PTA test results at 250, 500, 1000, 2000,4000 and $8000 \mathrm{KHz}$ in the study sample was $16,15,6,8,10$ and 7 respectively. Postoperative PTA value was significantly lower in all frequencies (paired $t$ test, $\mathrm{p}<.001$ ). 


\section{Tables}

1. Chart 1 - Pre and post intervention mean distribution

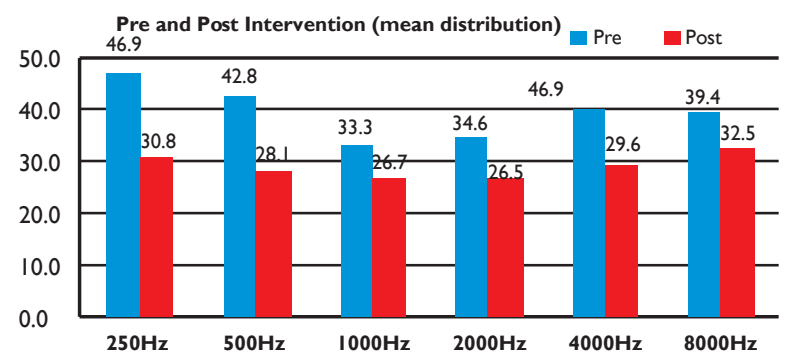

Chart 2 - Pre and post op test results at $500 \mathrm{~Hz}$

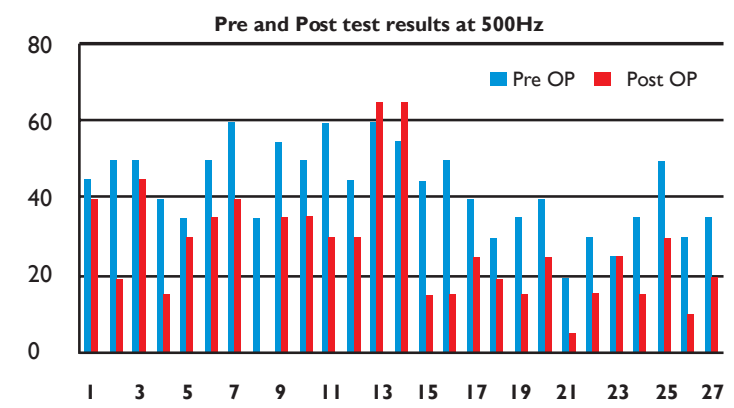

2. Chart 3 - Pre and post op test results at $250 \mathrm{~Hz}$

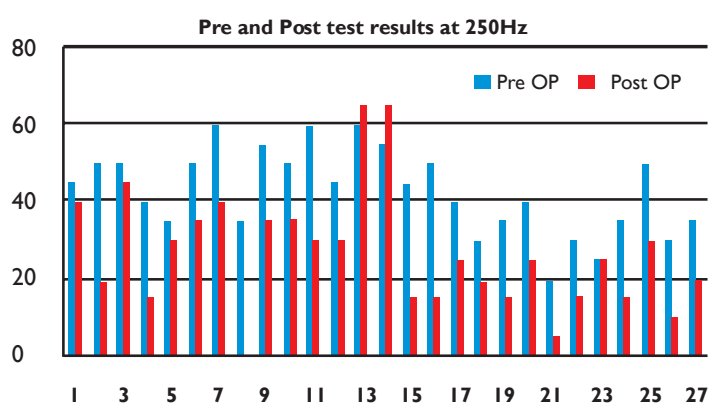

\section{Discussion}

Tympanic membrane perforation (safe type) is not rare among E.N.T. clinic patients. It interferes with patients day to day activities such as swimming and other water related activities. Further it interferes with hearing level (resulting conductive) deafness.

Repair of tympanic membrane defect in dry safe ear is a frequently performed procedure in E.N.T. setup. There are different methods and different types of graft materials used to repair the defects. The commonly accepted method is underlay myringoplasty using temporalis fascia graft (an autograft). Its success rate is about $80 \%$ in experienced hands and takes about $30-45 \mathrm{~min}$ for the procedure.

In our method it takes relatively shorter duration (about $15 \mathrm{~min}$ ) for the surgery and post-operative morbidity is less. Therefore patients can be dischargedearly and get back to routine day to day activity very early. Post aural incision is avoided and only has a $1 \mathrm{~cm}$ size scar in periumbilical region of the abdomen

The following problems were encountered in the study.

All the patients were prescribed 'Oxoferine' which is costly and is about Rs.1,500/- each.

For those who could not afford, one sample was divided among 3-4 patients.

Exposure of the liquid to sunlight during transfer and storage would have affected the quality.

Some patients were lost to follow-up.

All the cases that failed to achieve a complete closure of the perforation underwent formal myringoplasty subsequently.

\section{Conclusion / Key Messages}

Repair of TM defects with fat and tetrachlorodecaoxide resultsin significant improvement in hearing levels.

\section{References.}

1. Ringenberg J.C. (1962) Fat graft tympanoplasty. Laryngoscope 72, 188-192

2. Kelvin M. Kwong, Matthew M. Smith,James,M. Coticchia,International Journal of PediatricOtorhinolaryngology, Volume 76, Issue 8 , Pages 1098-1101, August 2012 
3. Dursun E, Dogru S, Gungor A, Cincik H, Poyrazoglu E, OzdemirT.,Comparison of paper-patch, fat, and perichondrium myringoplasty in repair of small tympanic membrane perforations.,Otolaryngology Head Neck Surg. 2008 Mar;138(3):353-6

4. Saliba, I. Hyaluronic acid fat graft myringoplasty: how we do it, Clinical Otolaryngology 33, 610-614

5. Konstantinidis.I, Malliari,H . Tsakiropoulou, Constantinidis J,Fat myringoplasty as an office based procedure2010,OtorhinolaryngologiaHead and Neck Surgery $42,25-28$

6.Dr.VikasSinhadr_sinhavikas@ yahoo.co.in,http://www.waent.org/ archives/2009/vol2-2/20091204-fatplug-myringoplasty/fat-plug.htm7

7. Elwood MJ, Pearson GCJ, Skippen HD, Jackson MS (1984). Alcohol, smoking, social and occupational factors in the aetiology of cancer of the oral cavity, phayrnx and larynx. Int J Cancer, 34, 603-12.

8. Kelvin M.Kwons, Matthew M. Smith, James M. CoticchiaFat graft myringoplasty using umbilical fat

Department of Otolaryngology-Head \&Neck Surgery, Wagne State University School of Medicine, Detroit, MI, United States

www.ijporlonline.com/article/SO1655876 (12)00245-5/abstract

9. Constantinids.I, H.Malliari,E. Tsakiropoulou, J.Fat myringoplasty as an office based procedure

$2^{\text {nd }}$ Academic ORL Department, Aristotal University, Papageorgious Hospital, Thessaloniki, Greece.

www.otorhinolaryngologia.gr/ issues/5Ci_42\%5CT42_p25-28.pdf
10. Gian Antonio Bertoli, MD; Marco Barbaro , MD; Valerio Giangrande, MD; GianlucaBava, MD; Elio De Seta, MD; Roberto Filipo, MDFat graft myringoplasty: An office procedure for the repair of small1. Perforations of the tympanic membrane

www.mediotol.org/MJOg/Bertoli.pdf

Corresponding Author:

Dr.UpulAbeysundara

Teaching Hospital, Anuradhapura, SriLanka.

Email-abeysundaraupul@yahoo.com 